\title{
Studies on the $\Lambda(1405)$ and the antikaon-nucleon interactions with the KLOE Drift Chamber
}

\section{O. Vázquez Doce*}

Excellence Cluster Universe, Technische Universität München, Garching, Germany

E-mail: oton.vazquez@universe-cluster.de

on behalf of the AMADEUS collaboration

\section{Curceanu}

INFN, Laboratori Nazionali di Frascati, Frascati (Roma), Italy

\section{K. Pischicchia}

INFN, Laboratori Nazionali di Frascati, Frascati (Roma), Italy

Museo Storico della Fisica e Centro Studi e Ricerche "Enrico Fermi", Roma, Italy

\section{A. Scordo}

INFN, Laboratori Nazionali di Frascati, Frascati (Roma), Italy

\section{Tucakovic}

INFN, Laboratori Nazionali di Frascati, Frascati (Roma), Italy

The strangeness sector of the non-perturbative regime of the low energy region of QCD is of capital importance for the understanding of the hadron interactions and structure, and its in-medium modifications. The kaon-nucleon interaction, and the study of the antikaon-nucleon potential in nuclear matter is the main ingredient for understanding how a state of kaon condensate could appear in conditions of extreme density (as in the core of the neutron stars) and its influence in the nuclear equation of state.

The studies here proposed aims to extract new information from the most accurate available data from kaonic absorption by light nuclei, with the interpretation of the results from the analysis of the KLOE experiment data, where low momentum and stopped kaons are absorbed in the various components of the spectrometer (being of special interest those produced in the solid ${ }^{12} \mathrm{C}$ of the drift chamber entrance wall and in the ${ }^{4} \mathrm{He}$ gas filling the chamber). A sample of $1.4 \mathrm{fb}^{-1}$ has been analyzed and output distributions for invariant masses, missing masses, momenta, etc., are available for different channels $(\Sigma \pi, \Lambda \mathrm{p}, \Lambda \pi)$ with almost full acceptance.

XV International Conference on Hadron Spectroscopy-Hadron 2013

4-8 November 2013

Nara, Japan

\footnotetext{
*Speaker.
} 


\section{Introduction}

The modification of the properties and interactions of the antikaon in the nuclear medium is currently being systematically studied from the theoretical and experimental point of view, with a renewed interest since the prediction of the existence of the bound kaonic clusters, where a $\mathrm{K}^{-}$is attached in a super-dense state (several times standard nuclear density). The prediction is based on a very deep attractive potential, thus allowing the formation of kaonic states with narrow width and considerably high binding energies, which would allow its detection via hadron spectroscopy. The approach with phenomenological optical potentials included in the original prediction was reiterated lately [1] and has been confirmed in the framework of the Skyrme model [2]. A different many-body calculation, taking into account the two-nucleon absorption process, leads to potentially non-observable states with small binding energies and large widths [3]. Higher bindings are also put into doubt by non perturbative coupled channels Chiral SU(3) dynamics [4], and 3-body Faddeev calculations predict deeply bound states only in heavy systems [5]. Recently more precise predictions on the value of the antikaon-nucleon potential in the nuclear environment [6] have been possible thanks to the successful finalization of the SIDDHARTA experiment [7]. From the experimental point of view, two main approaches have been used for studying the kaonic clusters: proton-proton and heavy ion collisions (FOPI [8], HADES [22]), and low momentum or stopped $\mathrm{K}^{-}$interactions in light nuclei (FINUDA [10] and KEK-PS E549 collaborations [11]). Data from not dedicated experiments are being re-analyzed as in the case of DISTO [12]. The interpretation of the absorption experiments results is far from being conclusive, and it requires an accurate description of the single and multi-nucleon absorption processes that a $\mathrm{K}^{-}$would undergo when interacting with light nuclei [13]. The correct interpretation requires a precise knowledge of the shape and characteristics of the single and multi-nucleon absorption processes, in order to decouple this type of background from an expected signal due to the formation of a bound state.

\section{The $\Lambda(1405)$}

The nature of the $\Lambda(1405)$ has been uncertain for decades. Despite the fact that this resonance has since then been observed in many experiments, its nature still remains unsettled. The three quark picture (uds) meets some difficulties to explain the observed $\Lambda(1405)$ mass. In the mesonbaryon picture the $\Lambda(1405)$ is viewed as a antiK-N quasi-bound $\mathrm{I}=0$ state, embedded in the $\Sigma \pi$ continuum, emerging in coupled-channel meson-baryon scattering models [14]. In the context of chiral unitary models [15] two poles emerge in the scattering amplitude (with $S=-1$ and $I=0$ in the neighborhood of the $\Lambda(1405)$ mass, both contributing to the final experimental invariant mass distribution. One pole is located at higher energy with a narrow width and is mainly coupled to the antiK-N channel, a second lower mass and broader pole is dominantly coupled to the $\Sigma \pi$ channel [16]. Since the resonance is always seen in the invariant mass spectrum of the $\Sigma \pi$ strong decay, the only chance to observe a higher mass component is by using the antiK-N production mechanism. Moreover the $\Sigma^{0} \pi^{0}$ channel, which is free from the $\mathrm{I}=1$ contribution and from the isospin interference term, turns to be the cleanest, and it is accessible to us in the absorptions detected in the KLOE data. In the case of the study of the $\Lambda(1405)$, the opportunity opened by the analysis of the KLOE data is even more obvious, since what experimentally has been obtained 
from absorption experiments (in bubble chamber [17] or emulsion [18]) has been produced only by identifying the charged decay channels, with the contamination from $\Sigma(1385)$ and moreover, with spectra always cut at the at-rest limit, which in the KLOE case will be complemented with the in-flight absorptions at a momentum of around $120 \mathrm{MeV} / \mathrm{c}$. Only non-absorption events present spectra in the $\Sigma^{0} \pi^{0}$ channel (from kaon [19] and pion beams [20], and lately with photo production, by CLAS [21], and pp collisions data, by HADES [22]), and with different line shapes.

\section{The KLOE data on $\mathrm{K}^{-}$absorptions}

Signals from $\mathrm{K}^{-}$absorption (at rest and with low momentum, lower than $127 \mathrm{MeV} / \mathrm{c}$, coming from the $\phi$ meson decay almost at rest) have been investigated with a sample of $70 \%$ of the statistic collected by the KLOE experiment in 2004-2005, which amounts to $1.4 \mathrm{fb}^{-1}$. The KLOE spectrometer [23] consists of a drift chamber (DC) or two meters of outer radius filled mainly with ${ }^{4} \mathrm{He}(90 \%)$, that is surrounded by an electromagnetic calorimeter (EMC) responsible for the photon detection. The detection of a $\Lambda$ hyperon through its decay into a proton and a negative pion (with a branching ratio of 64\%) was used as a tagging. The proton and pion tracks are identified with the DC and its momentum is measured with a precision better than $1 \%$ (in the case of the proton). The decay vertex is known with an error below $1 \mathrm{~mm}$. For decays inside the gas volume, the Lambda invariant mass is obtained with a resolution of $0.3 \mathrm{MeV} / \mathrm{c}^{2}$ and a value of $1115.723 \pm 0.003 \mathrm{MeV} / \mathrm{c}^{2}$ (error only statistical).

Analyzing the intersection of the $\Lambda$ and proton tracks the absorption vertex can be identified. The resolution in the $\Lambda \mathrm{p}$ invariant mass can be improved by improving the reconstruction of the proton four-vector, and the material where the absorption takes place can be determined more precisely. The final output of the $\Lambda$ p inclusive analysis can be seen in the left part of Fig. 1 for the ${ }^{4} \mathrm{He}$ selection, where the invariant mass distribution is shown. A prominent enhancement is seen in the low invariant mass. This part of the spectrum is expected to be populated mainly by events coming from the single nucleon absorption process, where the kaon interacts with one nucleon producing an hyperon-pion pair, the proton in the final state coming from the initial nucleus [24] (thus initially not participating in the interaction) or from the $\Sigma / \Lambda$ nuclear conversion process [25] that can take place in the residual nucleus. As explained before, the knowledge of the shape of the $\Lambda \mathrm{p}$ invariant mass for the single and multi-nucleon absorption processes is of great importance in the search for the kaonic bound clusters.

For the low invariant mass the predominant contribution comes from the mesonic channels, and can in fact be explored in more detail. Following the same procedure used for the identification of the proton correlated with the absorption vertex, we search for a third particle, in this case a negative pion. This is indeed found in approximately $1 / 6$ of the $\Lambda$ p events, and the invariant mass for this subsample is represented by the dashed line in Fig. 1 (left), with an arbitrary normalization, in order to be compared with the full spectrum. With the requirement of the presence of a pion track, the contribution from the high energy tail loses strength, when compared with the inclusive selection.

For the study of the $\Lambda(1405)$, the $\Sigma^{0} \pi^{0}$ decay channel has been analyzed. The selection of events proceeds after the identification of a $\Lambda$, with the identification of three photon clusters via 
the EMC. Three calorimeter hits not associated to any DC track, and in time with the lambda decay vertex, are searched for and selected on the base of a $\chi^{2}$ for the TOF and the reconstructed $\pi^{0}$ mass.

The obtained invariant mass for $\Sigma^{0} \pi^{0}$ is shown in the right part of Fig. 1 for events selected in ${ }^{12} \mathrm{C}$, as well as the cluster momentum. The mass distribution shows an excess of events above the kinematical limit for $\mathrm{K}^{-}$absorptions at rest. The kinematical limit is almost the same for absorptions at rest in carbon and helium ( $1416 \mathrm{MeV} / \mathrm{c}^{2}$ in ${ }^{12} \mathrm{C}$ and $1412 \mathrm{MeV} / \mathrm{c}^{2}$ in ${ }^{4} \mathrm{He}$ ) as it depends on the last nucleon binding energy which differs only by $4 \mathrm{MeV}$ for the two nuclei. Based on this, the mass spectra was split into in-flight and at rest candidate events. Both distributions show a structure with two components, with the lower momentum one correlated with high masses and vice versa.
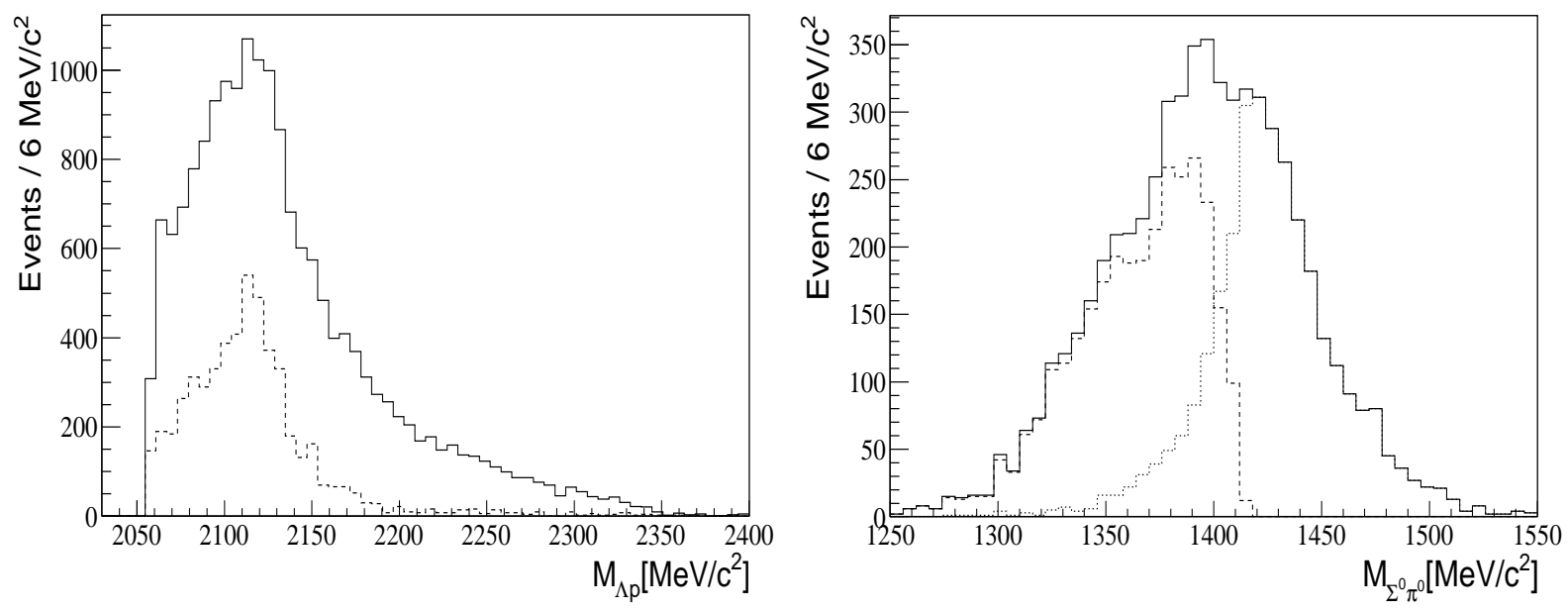

Figure 1: Left: $\Lambda \mathrm{p}$ invariant mass for the inclusive $\Lambda \mathrm{p}$ selection (continuous line) and for events with an extra $\pi^{-}$detected (dashed line). Right: $\Sigma^{0} \pi^{0}$ invariant mass (dashed and pointed line represent the at-rest and in-flight components respectively).

The interpretation of the data obtained with KLOE will allow to obtain a complete and firm result, with qualitative and quantitative outputs from $\mathrm{K}^{-}$absorption to be added to the pp and heavy ion collisions data.

It is clear that is very important to achieve a clear distinction between the in-flight and at-rest spectral shape of the $\Lambda(1405)$ to correctly interpret the results and compare the last to theoretical models, with a extraction of the resonant and non-resonant part, and characterization of the line shapes of the resonance. The specific theoretical predictions for the mass distributions of the $\Lambda(1405)$ can be directly compared with the results obtained and the two pole version of the theories. From the lambda-baryon channel analyses, the determination of the fraction single/multi nucleon absorption in ${ }^{4} \mathrm{He}$ and ${ }^{12} \mathrm{C}$ will be obtained, as well as the rates of nuclear $\Sigma / \Lambda$ conversion in for the given energy range. The results can give strength to models in which the interaction takes place with a free proton or to those with a bound proton, and can be related to the sub threshold modification of the anti-KN interaction. 


\section{References}

[1] Y. Akaishi and T. Yamazaki, Nucl. Phys. A 792, 229 (2007).

[2] T. Nishikawa and Y. Kondo, arXiv:0710.0948v2 [hep-hp] (2007).

[3] L. Tolos, A. Ramos and E. Oset, Phys. Rev. C 74, 015203 (2006).

[4] A. Dote, T. Hyodo and W. Weise, Phys. Rev. C 79, 014003 (2009).

[5] Y. Ikeda and T. Sato, Phys. Rev. C 79, 035201 (2009).

[6] Y. Ikeda, T. Hyodo and W. Weise, Nucl.Phys. A881, 98 (2012).

[7] M. Bazzi et al., Phys. Lett. B 704, 113-117 (2011).

[8] K. Suzuki et al., Nucl. Phys. A 827, 312C-314C (2012).

[9] G. Agakishiev et al., Phys. Lett. C 85, 035203 (2012).

[10] M. Maggiora et al., Nucl. Phys. A 835, 43-50 (2010).

[11] M. Agnello et al., Phys. Rev. Lett. 94, 919303 (2005).

[12] T. Suzuki et al., Mod. Phys. Lett. A 23, 2520-2523 (2008).

[13] V. K. Magas, E. Oset, A. Ramos, Phys. Rev. C 74, 025206 (2006).

[14] R. H. Dalitz, T. C. Wong, G. Rajasekaran, Phys.Rev. 153, 1617 (1967).

[15] E. Oset, A. Ramos, Nuclear Phys. A635, 99 (1998).

[16] Y. Ikeda, T. Hyodo, W. Weise, Nucl. Phys. A881, 98 (2012).

[17] D. Riley et al., Phys. Rev. D11, 3065 (1975).

[18] P. J. Carlson et al., Nuclear Phys. 74, 642 (1965).

[19] Prakhov, et al., Phys. Rev. C70, 034605 (2004).

[20] D. W. Thomas, A. Engler, H. E. Fisk, and R. W. Kraemer, Nucl. Phys. B56, 15 (1973).

[21] K. Moriya et al. (CLAS collaboration), Phys. Rev. C 87, 035206 (2013).

[22] G. Agakishiev et al. (HADES collaboration), arXiv:1208.0205v2 (2013).

[23] M. AdinoliňĄ et al., Nucl. Instr. Meth. A 488, 51-73 (2002).

[24] C. Vander Velde-Wilquet et al., Nucl. Phys. A 241, 511 (1974).

[25] R. Roosen et al., Nuov. Cim. 49A 2, 217 (1979). 